\title{
Highly Stretchable Two-Dimensional Auxetic Metamaterial Sheets Fabricated via Direct-Laser Cutting
}

\author{
Luke Mizzi $^{1 *}$, Enrico Salvati ${ }^{2}$, Andrea Spaggiari ${ }^{1}$, Jin-Chong Tan ${ }^{3}$, Alexander M. Korsunsky ${ }^{2}$ \\ ${ }^{1}$ Department of Engineering Sciences and Methods, University of Modena and Reggio Emilia, Reggio Emilia, \\ Italy \\ ${ }^{2}$ MBLEM, Department of Engineering Science, University of Oxford, OX1 3PJ Oxford, United Kingdom \\ ${ }^{3}$ Multifunctional Materials \& Composites (MMC) Laboratory, Department of Engineering Science, University of \\ Oxford, OX1 3PJ Oxford, United Kingdom \\ *corresponding author email: luke.mizzi@unimore.it
}

\begin{abstract}
The design and production of multifunctional materials possessing tailored mechanical properties and specialized characteristics is a major theme in modern materials science, particularly for implementation in high-end applications in the biomedical and electronics industry. In this work, a number of metamaterials with perforated architectures possessing the ability to exhibit a plethora of 2D auxetic responses with negative Poisson's ratios ranging from quasi-zero to large negative values (lower than -3.5), stiffnesses, stretchability and surface coverage properties were manufactured. These systems were produced through the introduction of microstructural cuts in a rubber sheet using direct laser cutting, and analysed using a dual approach involving experimental tests and Finite Element Analysis. In addition to examining the mechanical properties of the perforated metamaterials, the influence of edge effects and material thickness on the deformation behaviour of these systems were investigated, with re-entrant systems shown to possess anomalous deformation profiles which are heavily dominated by the boundary regions. These findings highlight the effectiveness of this method for the fabrication of auxetic metamaterial sheets as well as the large variety of mechanical properties, deformation mechanisms and load responses which may be obtained through what may be effectively described as simply the introduction of patterned cuts in a thin sheet.
\end{abstract}

Keywords: Mechanical Metamaterials, 2D Auxetics, Perforated Systems, Direct Laser Cutting

\section{Introduction}

Auxetic mechanical metamaterials are systems which possess the unusual property of having a negative Poisson's ratio[1]. This property arises primarily as a result of the structure of the system rather than its chemical and material composition and thus the mechanisms which 
impart this characteristic are scale independent. In fact, auxeticity has been reported in a number of systems ranging from the macro-scale to the micro- and nano-levels. Auxetic metamaterials have attracted a great deal of interest not only due to their negative Poisson's ratio, but also for the variety of additional accompanying characteristics which include the ability to undergo synclastic curvature[2] and high indentation resistance[3]. These properties make these systems applicable for a wide range of uses, particularly in the biomedical[4][5][6][7], personal protection[8], aviation[9][10] and electronics sectors[11][12].

A number of rapid prototyping techniques have been suggested and implemented for the manufacture of auxetic metamaterials ranging from additive manufacturing methods such as extrusion-based and stereolithography 3D printing [13][14][15][16][17], to molding[18] and laser lithography[19][20][21]. These methods allow for the production of mechanical metamaterials on a wide range of scales with their suitability for the specific fabrication of different metamaterial types depends on a variety of factors such as desired resolution, material type, scale, geometric characteristics and required properties. In this work, we explore the effectiveness and viability of using direct laser cutting as a method for the design of thin sheetlike 2D perforated auxetic metamaterials. These perforated auxetic systems are based on the concept that the introduction of strategically placed cuts and/or perforations within a suitable thick sheet of material possessing a positive Poisson's ratio can transform the system into exhibiting a tailor-made negative Poisson's ratio[22,23,32-35,24-31][36][37][38]. In particular, this work will focus on auxetic systems produced through the introduction of slit perforations, which involve only the introduction of a cut rather than the actual removal of a chunk of material from the sheet, a factor which potentially makes laser cutting an ideal candidate for rapid production of such structures. Although several slit perforation patterns have been proposed which may be used to design thin-sheet kirigami structures capable of exhibiting auxetic behaviour, the deformation of these systems is typically dominated by outof-plane deformations [39-41] which generally results in an overall much less negative Poisson's ratio than that predicted for solely in-plane deformation. One way through which this problem may be circumvented is to produce systems with very large out-of-plane thickness in order to minimise this effect. However, this solution is inadmissible for thin sheet-like systems requiring large in-plane auxetic behaviour and thus in these cases it is imperative to counteract this problem through smart design of the metamaterial system.

In view of this, in this work twelve mechanical metamaterial geometries belonging to four distinct classes of auxetic structures, were produced through the introduction of microstructural 
cuts on a $1 \mathrm{~mm}$ rubber sheet using direct laser cutting. These geometries range from systems which are numerically predicted to exhibit giant auxetic in-plane behaviour (i.e. Poisson's ratios lower than -3) to systems which show a Poisson's ratio of zero and are produced using perforations with dimensions which are relative to the overall out-of-plane thickness of the sheet. The in-plane mechanical properties of these systems were investigated using a microtensile loading device and digital imaging correlation (DIC) studies and compared with the predictions of non-linear Finite Element simulations on corresponding structures. Furthermore, the influence of boundary effects and out-of-plane deformations on the mechanical properties of these systems were also studied.

\section{Design of Perforated Metamaterial Systems}

The twelve geometries chosen for study and the parameters used to define the architectures are depicted in Figure 1 below. These systems were produced from a $1 \mathrm{~mm}$ rubber through the introduction of $300 \mu \mathrm{m}$ cuts and the resulting in-plane repeating unit dimensions ranged from as low as $3.5 \mathrm{~mm}$ up to $10 \mathrm{~mm}$, depending on the metamaterial geometry in question. Many of these geometries have been predicted, through numerical simulations conducted under planestress and periodic boundary conditions, to possess the potential to exhibit an extremely wide range of negative Poisson's ratios. These geometries represent almost all classes of 2D auxetic mechanisms and thus can be considered as ideal candidates for an experimental analysis on their effectiveness when implemented in thin sheet systems. Besides allowing one to experimentally evaluate the in-plane auxetic capabilities of these systems, these studies are also expected to give a clear picture of other important characteristics such as boundary effects, outof-plane deformation and defects on the performance of each individual perforation geometry, which are essential for ensuring metamaterial functionality.

These chosen geometries may be classified into four main groups. The first group comprising Architectures I-III is created through the introduction of straight-line slits organized into two alternating sets. In Architecture I, the two sets of slits are equally sized and are aligned in a perpendicular orientation relative to each other. In Architecture II, the slits also possess a perpendicular alternating orientation, but they are not equal in length, while in Architecture III, the slits are also unequally sized and, in addition, have a variable relative orientation which is defined by parameter $\kappa$. These systems have been previously studied from a numerical and theoretical perspective and are predicted to exhibit deformation behaviour similar to the 
rotating quadrilateral mechanism[28]; more specifically the rotating squares[42], rotating Type I rectangles[43] and rotating Type I $\alpha$ parallelograms[44] mechanism for Architectures I-III respectively.

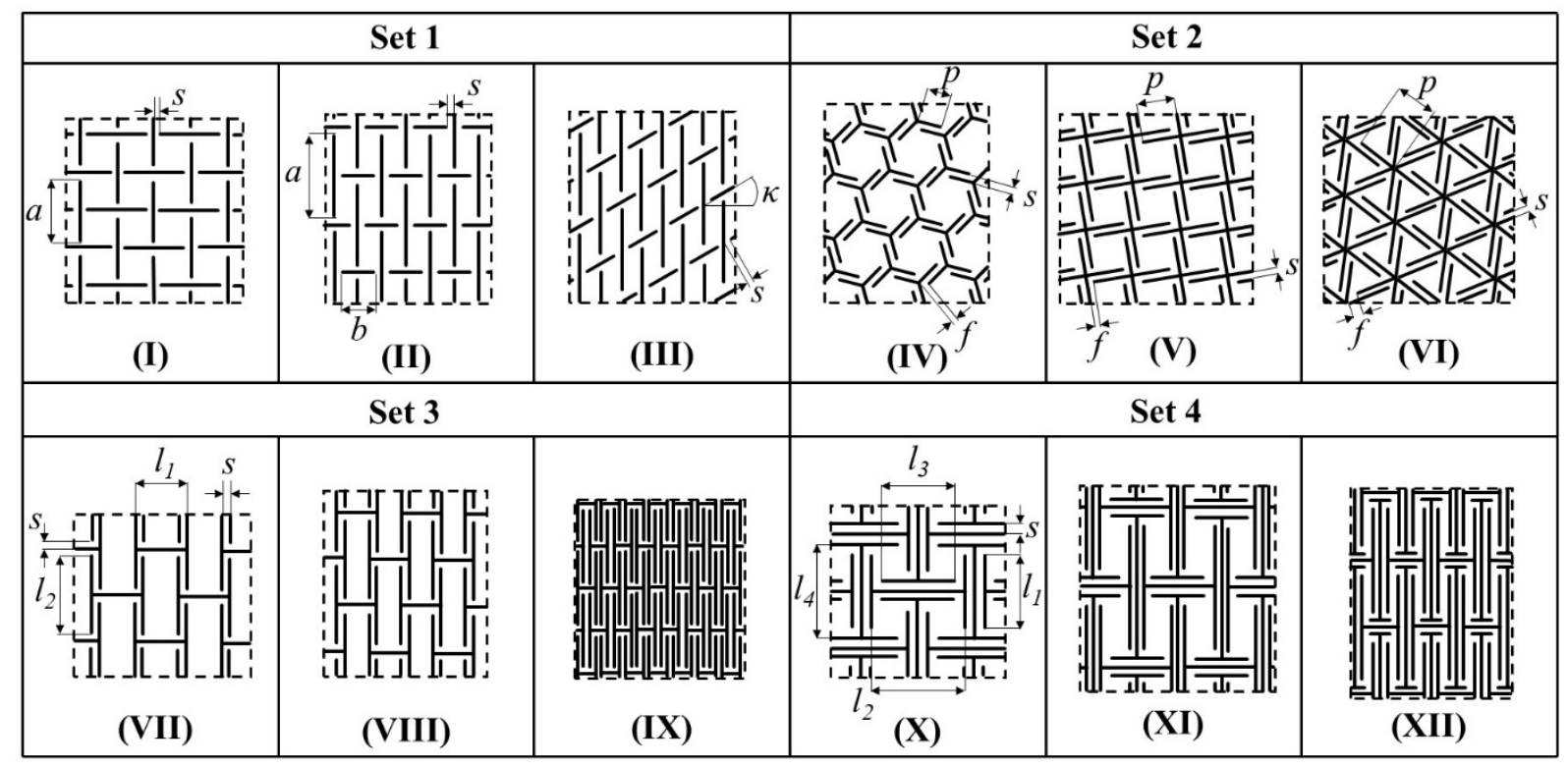

Figure 1: Diagram depicting the twelve architectures studied here and the parameters used to define the slit perforations. Note that Set 1 , Set 3 and Set 4 architectures may each be defined by the same set of parameters. In each case the perforations (marked by the black lines) had a thickness, $t, 0 f 0.3 \mathrm{~mm}$.

The second set of systems (Architectures IV-VI) consists of slit perforated systems designed in a star-shaped manner. These systems may be considered as the slit perforated counterparts of the area perforated three-star, four-star and six-star systems[16] which are designed to mimic the behaviour of hexachiral[45,46][47], tetrachiral[48][47][49] and trichiral[47][50] honeycombs with polygonal rotating units respectively. However, since in these cases the triangular section of the 'star' perforation is now defined solely the length, $h$, and thickness of the slit perforation, $t$, an additional parameter (shown in Figure 1) is required to define the shift of the perforations with respect to one another, $f$. The inclusion of this additional parameter was necessitated by the fact that unlike their area perforated counterparts (where this parameter was assumed to be zero), aligning adjacent perforations directly with one another results in a fragmented system for a considerable number of geometries (indeed, this is the case for all four and six-pointed star slit perforated systems) and thus does not result in a viable final structure.

The last two sets involve systems made from ' $I$ '-shaped slits[28][51]. In the third set, which comprises Architectures VII-IX, the slits are all aligned in the same manner. Three different sets of $l_{1}$ and $l_{2}$ values were used to define the systems and this resulted in Architecture VII having a thick column-like block of material, Architecture VIII having a medium sized block 
of material and Architecture IX being made up entirely of equally sized ligaments. These systems were designed to mimic the re-entrant mechanism, either in the form of the so-called 'fibril and nodule' mechanism or the hexagonal re-entrant honeycomb [52-55]. Finally, in the fourth set (Architectures X-XII), the systems are designed to deform similar to various antitetrachiral honeycombs[56][57] made from polygonal rotating units. These systems consist of two sets of 'I'-shaped slits aligned in a perpendicular orientation and are meant to mimic a regular anti-tetrachiral honeycomb (Architecture $\mathbf{X}$ ) and two irregular anti-tetrachiral systems (Architectures XI and XII).

\section{Methodology}

The parameters used to produce these twelve 2D architectures are listed in Table 1. The patterned perforations were cut using a Universal ${ }^{\circledR}$ XLS10 laser cutter using a single pass pathway from a piece of black rubber with a thickness, $d$, of $1 \mathrm{~mm}$. Following cutting, the samples were cleaned under sonication in order to remove any residual rubber fragments. The stress-strain plot of the base material is provided in Section 1 of the Supplementary Information. In each case, the thickness of the slit, $t$, was set to $300 \mu \mathrm{m}$, the chosen spot-size of the laser (see Figure 2a,b).

Table 1: List of dimensions used to design the slit perforated systems based on the parameters shown in Figure 1.

\begin{tabular}{|c|c|c|c|c|c|c|c|c|}
\hline \multicolumn{2}{|c|}{ Set 1 - Rotating Quadrilateral Systems } \\
\hline Architecture & $\begin{array}{c}a \\
(\mathrm{~mm})\end{array}$ & $\begin{array}{c}b \\
(\mathrm{~mm})\end{array}$ & $\kappa$ & $\begin{array}{c}s \\
(\mathrm{~mm})\end{array}$ & $\begin{array}{c}t \\
(\mathrm{~mm})\end{array}$ & $\begin{array}{c}\text { No. of } \\
\text { Repeating } \\
\text { Units in } x\end{array}$ & $\begin{array}{c}\text { No. of } \\
\text { Repeating } \\
\text { Units in } y\end{array}$ & $\begin{array}{c}\text { Gauge Length } \\
(\mathrm{mm})\end{array}$ \\
\hline I & 5.0 & 5.0 & $0^{\circ}$ & 0.4 & 0.3 & 5 & 7 & 44.8 \\
\hline II & 7.7 & 3.0 & $0^{\circ}$ & 0.4 & 0.3 & 7 & 5 & 45.5 \\
\hline III & 7.7 & 3.0 & $30^{\circ}$ & 0.4 & 0.3 & 7 & 5 & 45.7 \\
\hline Set 2 - Star Perforated Systems & $p$ & $f$ & $s$ & $t$ & $\begin{array}{c}\text { No. of } \\
\text { Repeating }\end{array}$ & $\begin{array}{c}\text { No. of } \\
\text { Repeating }\end{array}$ & $\begin{array}{c}\text { Gauge Length } \\
\text { (mm) }\end{array}$ \\
\hline Architecture & $\begin{array}{c}p \\
(\mathrm{~mm})\end{array}$ & $(\mathrm{mm})$ & $(\mathrm{mm})$ & $(\mathrm{mm})$ & $\begin{array}{c}\text { Units in } x \\
\text { Units in } y\end{array}$ & 45.7 \\
\hline IV & 3.2 & 0.5 & 0.4 & 0.3 & 6 & 6 & 45.2 \\
\hline
\end{tabular}




\begin{tabular}{|c|c|c|c|c|c|c|c|c|c|}
\hline Architecture & \multicolumn{2}{|c|}{$\begin{array}{c}l_{1} \\
(\mathrm{~mm})\end{array}$} & $\begin{array}{c}l_{2} \\
(\mathrm{~mm})\end{array}$ & \multicolumn{2}{|c|}{$\begin{array}{c}S \\
(\mathrm{~mm})\end{array}$} & $\begin{array}{c}t \\
(\mathrm{~mm})\end{array}$ & $\begin{array}{c}\text { No. of } \\
\text { Repeating } \\
\text { Units in } x\end{array}$ & $\begin{array}{c}\text { No. of } \\
\text { Repeating } \\
\text { Units in } y\end{array}$ & $\begin{array}{l}\text { Gauge Length } \\
(\mathrm{mm})\end{array}$ \\
\hline VII & \multicolumn{2}{|l|}{5.0} & 7.5 & \multicolumn{2}{|l|}{0.4} & 0.3 & 4 & 5 & 44.5 \\
\hline VIII & \multicolumn{2}{|l|}{3.0} & 6.0 & \multicolumn{2}{|c|}{0.4} & 0.3 & 7 & 6 & 44.4 \\
\hline IX & \multicolumn{2}{|l|}{2.3} & 7.5 & \multicolumn{2}{|c|}{0.4} & 0.3 & 11 & 5 & 44.5 \\
\hline \multicolumn{10}{|c|}{ Set 4 -Anti-Tetrachiral Systems } \\
\hline Architecture & $\begin{array}{c}l_{1} \\
(\mathrm{~mm})\end{array}$ & $\begin{array}{c}l_{2} \\
(\mathrm{~mm})\end{array}$ & $\begin{array}{c}l_{3} \\
(\mathrm{~mm})\end{array}$ & $\begin{array}{c}l_{4} \\
(\mathrm{~mm})\end{array}$ & $\begin{array}{c}s \\
(\mathrm{~mm})\end{array}$ & $\begin{array}{c}t \\
(\mathrm{~mm})\end{array}$ & $\begin{array}{c}\text { No. of } \\
\text { Repeating } \\
\text { Units in } x\end{array}$ & $\begin{array}{c}\text { No. of } \\
\text { Repeating } \\
\text { Units in } y\end{array}$ & $\begin{array}{l}\text { Gauge Length } \\
\text { (mm) }\end{array}$ \\
\hline $\mathbf{X}$ & 4.8 & 6.2 & 4.8 & 6.2 & 0.4 & 0.3 & 5 & 6 & 45.6 \\
\hline XI & 8.6 & 5 & 3.6 & 10 & 0.4 & 0.3 & 5 & 4 & 44.4 \\
\hline XII & 8.6 & 3 & 1.6 & 10 & 0.4 & 0.3 & 7 & 4 & 44.4 \\
\hline
\end{tabular}
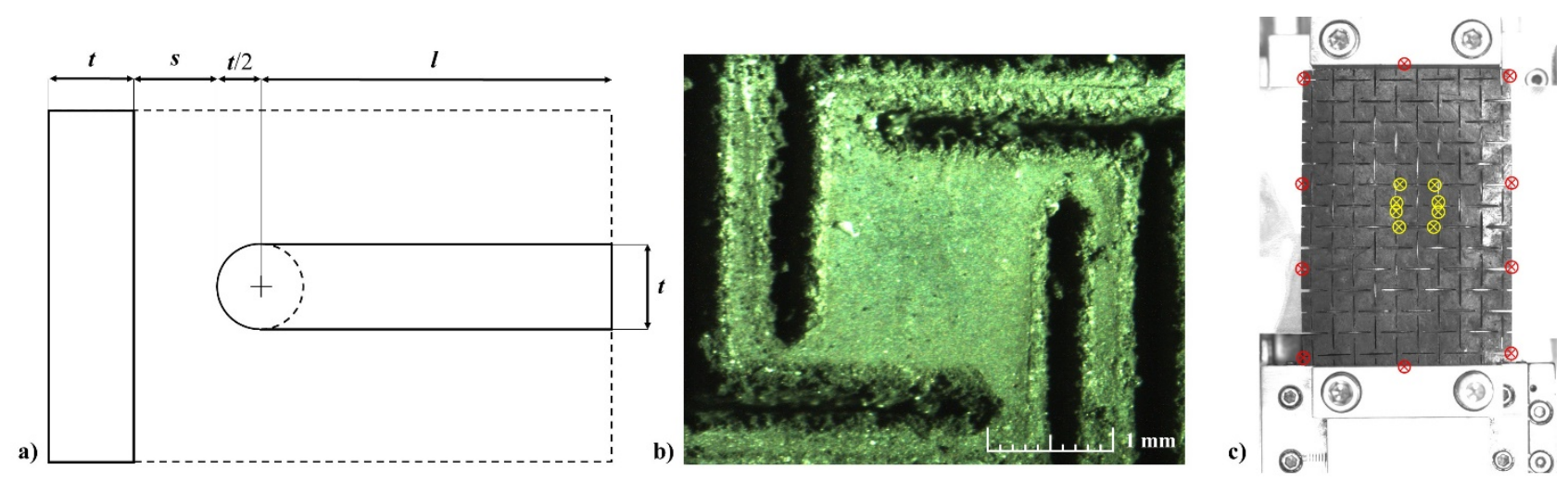

Figure 2: a) Schematic showing the actual dimensions of the slit perforations including the laser spot-size, $t$. The slit length, $l$, indicated in the diagram is an arbitrary term which represents the slit length parameters, $a, b, p, l_{1}, l_{2}, l_{3}$ and $l_{4}$ shown in Table 1 . As one may observe, the ends of the slits are circular and have a radius of $t / 2$. The separation between slits, $s$, is defined as the actual distance/length of material between the slits, including the slit thickness factor shown above. b) Image of Architecture $X$ under a stereomicroscope and c) image showing the 18 points from which the DIC measurements were taken for Architecture I; the yellow markers indicate the points on the central repeating unit while the red markers represent the points of the overall unit cell.

Since these systems possess an extremely low effective Young's modulus, a Deben ${ }^{\circledR}$ Micro tensile loading device with a $20 \mathrm{~N}$ load cell was used to measure the force required to apply a uniaxial tensile strain. The systems were loaded on the tensile loading device as shown in Figure $2 \mathbf{c}$ and an elongation of $10 \mathrm{~mm}$ (the maximum allowed by the tensile loading device) was applied to the architectures at a displacement rate of $1.5 \mathrm{~mm} / \mathrm{s}$. This corresponds to an overall tensile strain of roughly $20 \%$. In order to analyse the Poisson's ratio of these systems, the deformation was recorded and analysed using Digital Imaging Correlation (DIC) implemented in Matlab. The systems were analysed in terms of two aspects; i) the deformation of the central unit cell of each individual geometry and ii) the overall deformation of the entire 
system. In the former case, 8 markers denoting the central repeating unit of the geometry were tracked and used to find the strain in the $x$ - and $y$-directions as shown in Figure 11c (marked in yellow), while in the latter case an additional 10 points marking the external boundaries of the entire sample were tracked in order to obtain the average overall strains in the axial and transverse directions (marked in red). Further information on the DIC method is presented in Section 2 of the Supplementary Information.

In order to thoroughly analyse and evaluate the experimental Poisson's ratio plots obtained for these systems, non-linear simulations on equivalent periodic and finite systems were conducted for comparison. In the case of the infinite systems, the periodic boundary conditions were implemented using constraint equations on the edge nodes of the system, which was fixed to remain aligned along the $y$-axis. For the finite systems, the systems were designed and constrained in a similar method to that used in the experimental tests. Further simulation details are shown in Section 3 of the Supplementary Information. Also, similarly to the experimental prototypes, the Poisson's ratio of these systems was measured in two ways; i) from a central representative unit cell and ii) for the entire system overall, with readings taken from equivalent regions to the DIC measurements from the experimental prototypes. Since the main aim of this work was to analyse the in-plane deformation of these systems, the systems were simulated under plane-stress conditions using the PLANE183 element in ANSYS16 and using the material properties of the black rubber base material, with a Poisson's ratio of 0.49 . A hyperelastic 5-parameter Mooney-Rivlin model was fitted to the experimental stress strain data and used to obtain the parameters used to implement this model. Further information on the material properties are presented in Section 1 of the Supplementary Information. A 3D simulation using SOLID186 elements was also conducted in the case of one system which was shown to undergo significant out-of-plane deformation. In this specific case, periodicity was enforced only in the $x$ - and $y$-directions. Further details of this case are provided in Section 4 of the Supplementary Information.

\section{Results and Analysis}

In order to analyse the results obtained for each laser cut architecture in the context of the targeted deformation mechanism which it is meant to emulate, the results for each set of architectures are first divided and discussed in separate sections. The results involve a comparison of the experimental results compared with the corresponding planar Finite Element 
simulations on a finite system and on an infinite system using periodic boundary conditions. This is followed by an overall discussion of all the findings afterwards. Videos showing the experimental and simulations conducted in this study are presented in ANIMS01-38 as Supplementary Information.

$\underline{\text { Set } 1}$

As shown in the plots in Figure 3, all three architectures in this set exhibited a significantly negative Poisson's ratio, both experimentally and from the numerical simulations, with Architectures II and III showing a Poisson's ratio of less than -1. In addition, as one may also observe from the diagrams, the targeted rotating mechanism was more or less achieved in all systems. Animations showing the full deformation of the three architectures are presented in the ANIM01-09 and stress strain plots are presented in Section 5 in the Supplementary Information. 

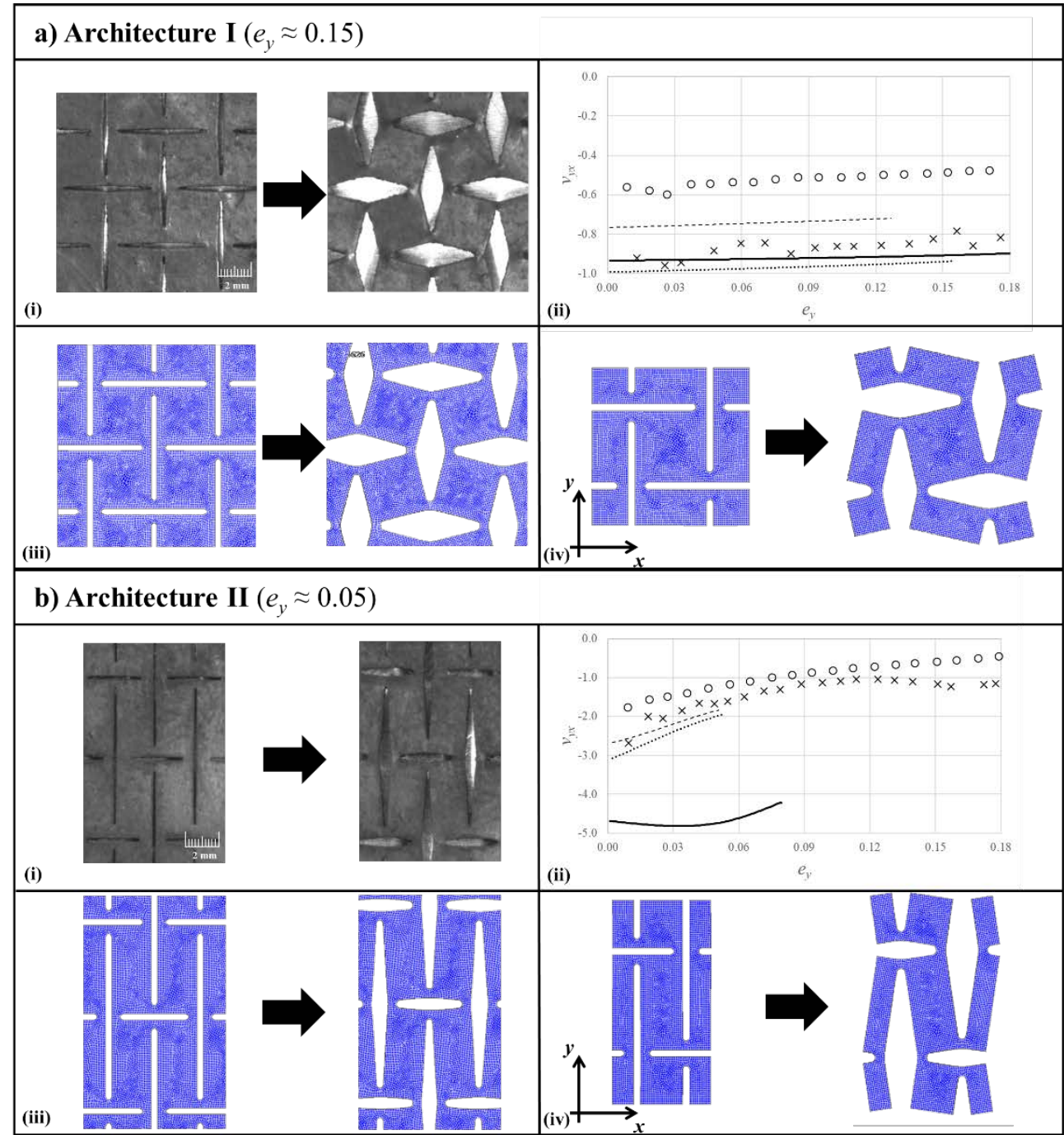

c) Architecture III $\left(e_{y} \approx 0.05\right)$

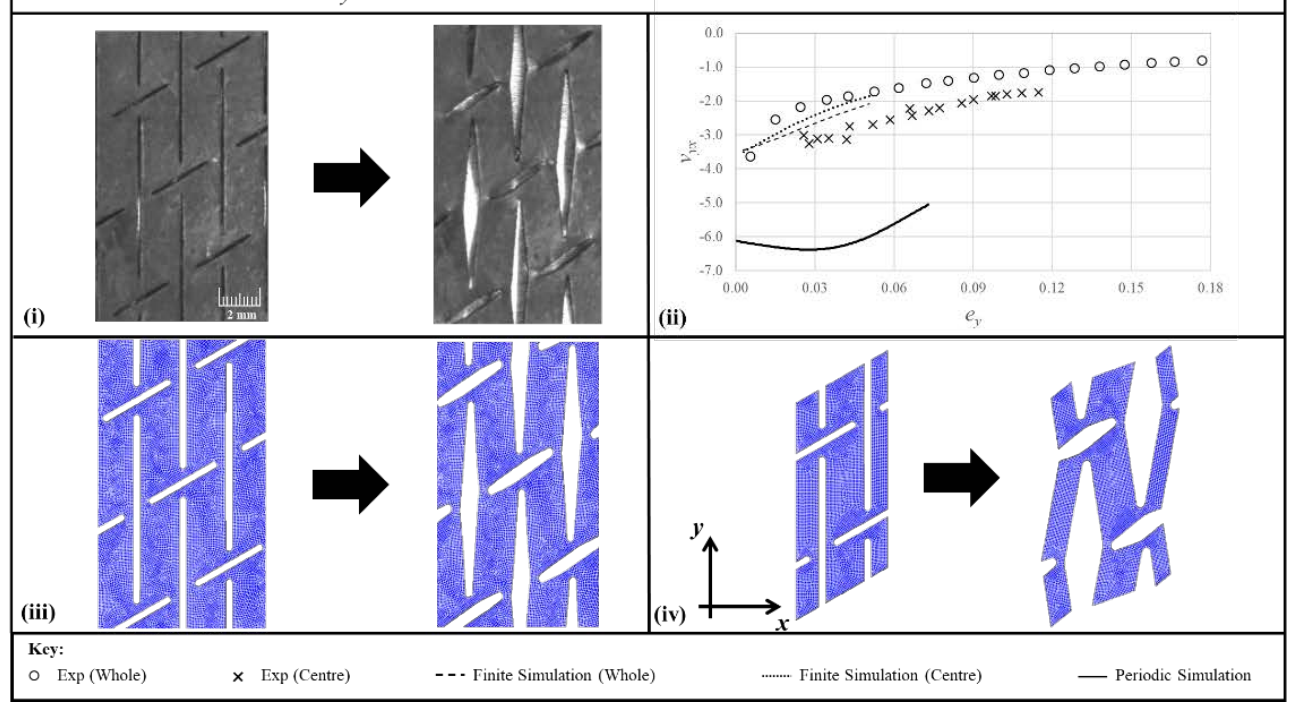

Figure 3: Diagram showing the central regions of the Set 1 (i) experimental and (iii) corresponding Finite Element simulation and (iv) the full periodic simulation for Architectures (a) I, (b) II and (c) III. The engineering Poisson's ratio, $v_{y x}$, vs engineering strain, $e_{y}$, plots for each system up to $18 \%$ strain as measured from the boundary and central regions of each sample are presented in (ii). Videos of experimental tests and simulations for these systems are presented in ANIM01-09. 
In each case, the measurements for the overall system yielded less negative Poisson's ratios than the ones taken from the centre. Furthermore, the central units experienced a significantly lower tensile strain in comparison to the overall systems, with the majority of deformation being observed in the boundary middle regions as shown in Figure 4. This behaviour was observed for both the simulated and experimental systems and may be explained by the fact that at the middle boundaries, the rotating units are the least constrained and hence they undergo the largest rotations. On the other hand, as expected, the regions close the clamps show the least rotating deformation. As a result of this deformation gradient, the central unit cell experiences a lower tensile strain than the overall system.

Architecture I shows the least difference between simulations and experiment, with the Poisson's ratio being almost constant between values in the range of -0.80 and -0.97 for the central unit cell. In fact, as one may observe from Figure 3a, the deformations shown are almost identical in all three cases. On the other hand, for Architectures II and III, while the finite simulations and experimental results are similar, the plots for the periodic simulations are significantly different; predicting in both cases a much more negative Poisson's ratio. This suggests that the effect of localized stretching at the 'joint' regions, which is present to a certain extent in all rotating unit systems made from perforated sheets, is exacerbated by the edge constraints on the system in the experimental tests and finite simulations and thus results in a less negative Poisson's ratio. In fact, as one may observe from Figure 3c(iv), in its free state, Architecture III has a non-zero shear coupling coefficient and hence a tendency to undergo shear deformations which are restricted by the clamping method used.
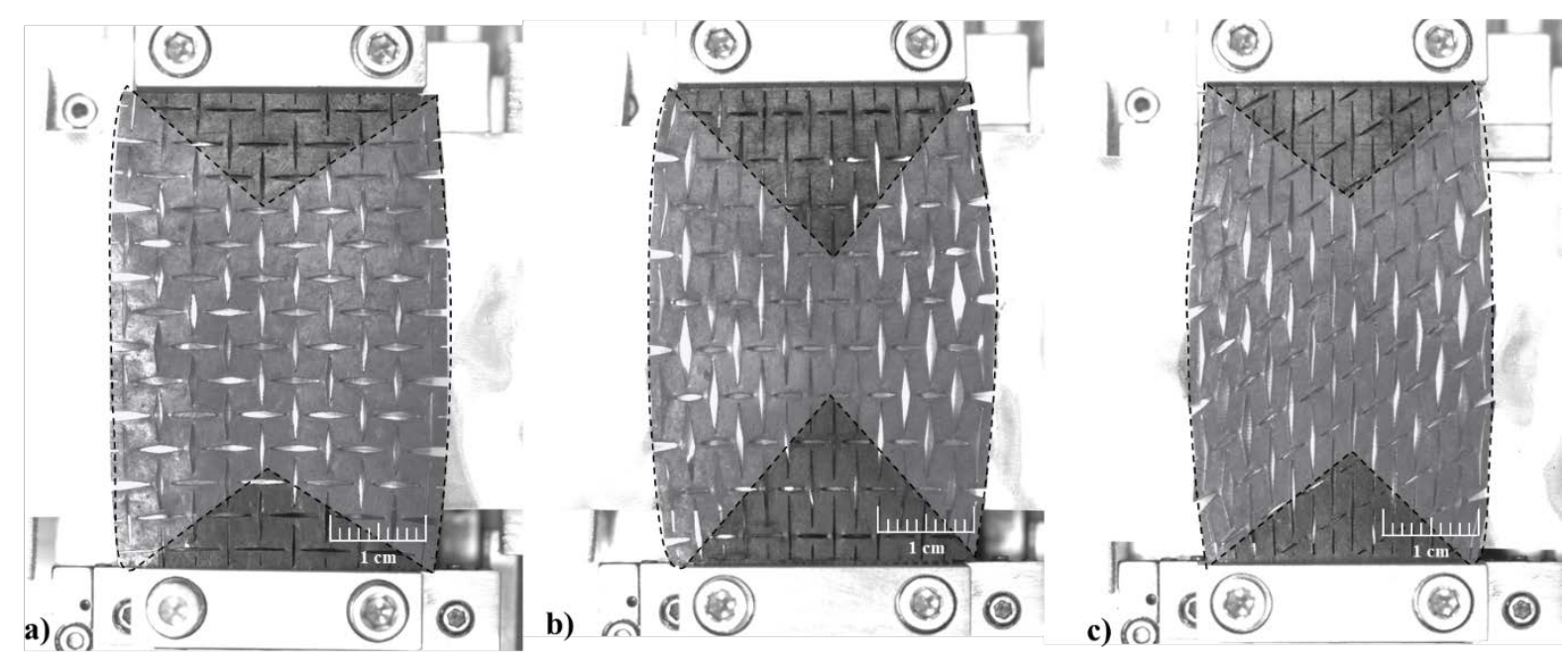

Figure 4: Diagrams showing the deformation gradient observed in Architectures a) I, b) II and c) III. The grey areas are used to highlight the regions exhibiting a relatively higher deformation. 
Moreover, as one may observe from Figure 3, the plots for the finite and periodic simulations did not reach the same level of strain as the experimental points. This is due to the fact that the simulations could not converge beyond a certain point because the rotating mechanism was close to reaching its point of maximum deformation. This was to be expected since previous analytical models on idealized rotating systems predict this phenomenon and it is well known that the chosen rotating rectangle and parallelogram geometries which Architectures II and III are meant to mimic have a relative low strain threshold in comparison to Architecture I (the rotating square mechanism). In fact, it was observed in the experimental tests that after a certain strain threshold ( $c a .4-5 \%$ and roughly equivalent to the corresponding simulations' failure point) Architectures II and III did not remain planar and started to undergo out-ofplane deformations in the form of waves parallel with the loading direction. The crests and throughs of the waves coincided directly with the vertically arranged slits (i.e. the ones defined by $l_{1}$ ) and this appears to indicate that material stretching is concentrated primarily at joint regions above and below the slits. Furthermore, at this point rotation of the subunits also ceased and the system started once again to contract, hence the lowering of the auxeticity of the systems at high strain. This behaviour can be visually observed in ANIM04 and ANIM07. In the planar finite element simulations, a similar scenario also occurs, however only in-plane stretching occurs due to the inherent planarity constraint. A similar effect was also observed for Architecture I, although not so pronounced and at a much high strain value. However, despite these limitations, both Architectures II and III still showed an initial experimentally measured Poisson's ratio in the regions of -2 and -3 respectively, highlighting the ability of this perforation patterning to achieve giant auxetic behaviour.

\section{$\underline{\text { Set } 2}$}

Set 2 architectures show the least discrepancies between the two sets of simulations and the experimental tests out of all the architectures investigated here. All systems show a near constant Poisson's ratio over an 18\% tensile strain range and these systems showed minimal out-of-plane deformations, even at high strains. The Poisson's ratios and deformed forms of these systems at 12\% engineering strain are presented in Figure 5, while videos of the tensile tests may be found in the Supplementary Information ANIM10-18. 


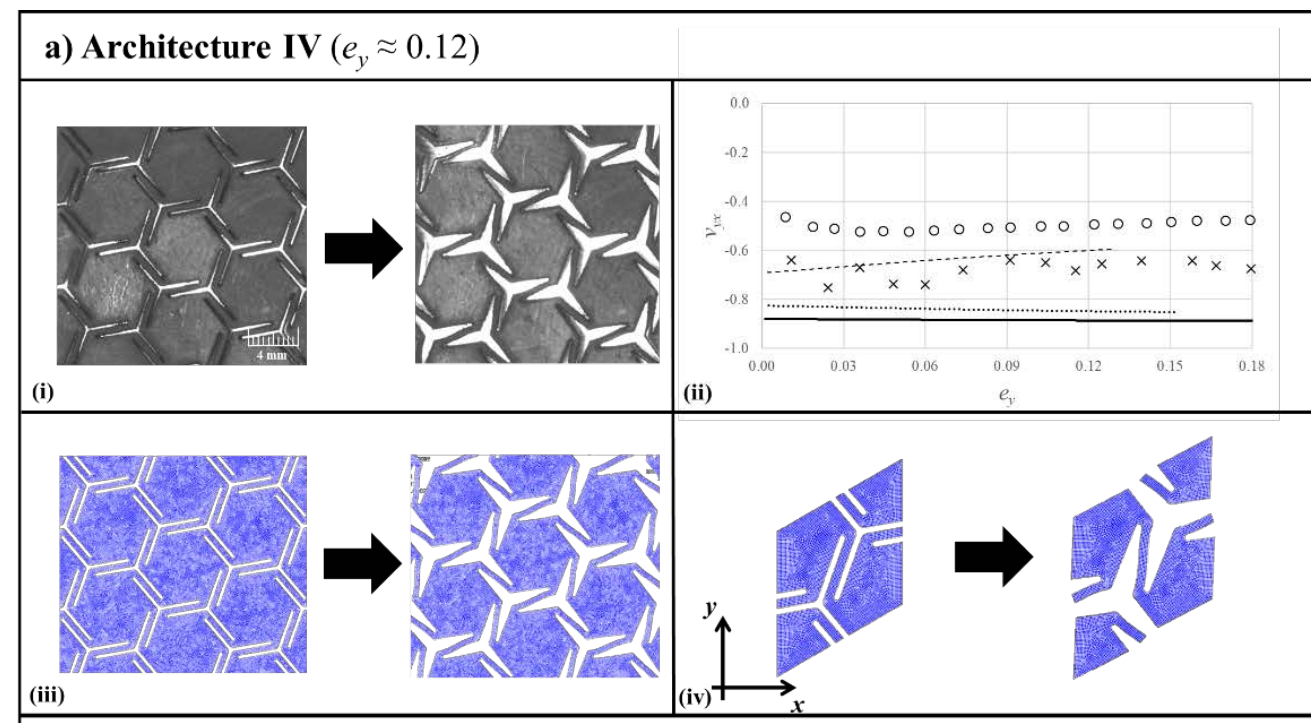

b) Architecture $\mathbf{V}\left(e_{y} \approx 0.12\right)$
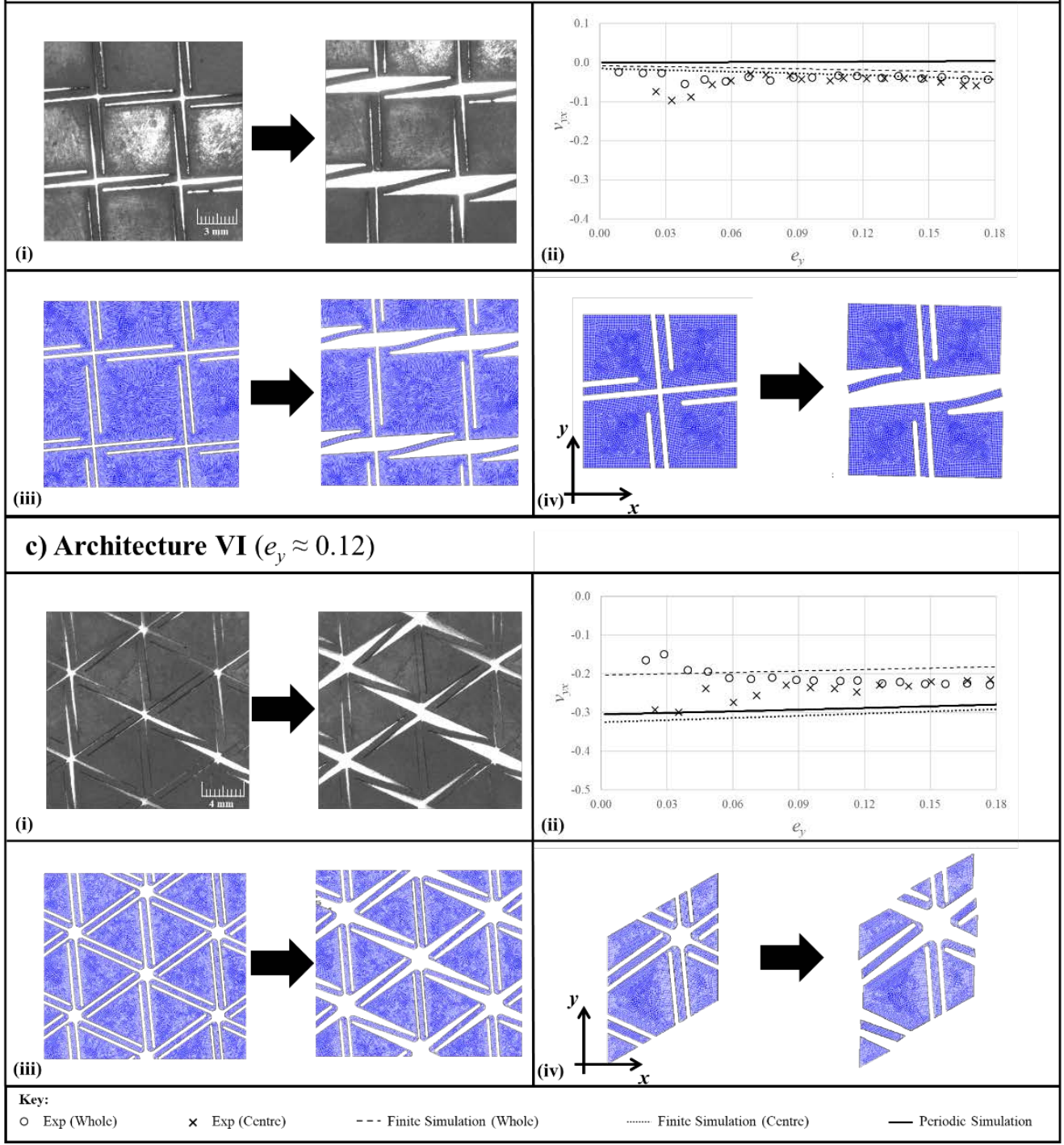

Figure 5: Diagram showing the central regions of the Set 2 (i) experimental and (iii) corresponding Finite Element simulation and (iv) the full periodic simulation for Architectures (a) IV, (b) V and (c) VI. The engineering Poisson's ratio, $v_{y x}$, vs engineering strain, $e_{y}$, plots for each system up to $18 \%$ strain as measured from the boundary and central regions of each sample are presented in (ii). Videos of experimental tests and simulations for these systems are presented in ANIM10-18. 
As evident from Figure 5, Architecture IV deformed in a manner similar to a hexachiral system made up of hexagonal rotating unit. This architecture is predicted to possess a nearconstant Poisson's ratio of $c a$. -0.85 from both the periodic and finite FE simulations while the value obtained from the experimental tests is slightly lower at $c a$. -0.7 . This behaviour is similar to that observed for the corresponding area perforated tri-star systems [16] and the difference in Poisson's ratio may be attributed to imperfections in the experimental systems resulting in slightly thicker ligaments that reduce the auxeticity of the system due to their resistance to flexural deformations[58]. The effect of boundary elements on the overall deformation is also quite low in this system with the Poisson's ratio decreasing by only ca. 0.2 for both the finite simulation and the experimental test when the Poisson's ratio of the whole system is measured. In fact, as one may observe from ANIM10 and ANIM11, the deformation gradient is more uniform than in the case of Set 1 systems, with the middle boundaries and central regions of the system showing more or less similar observable levels of deformation.

Architecture $\mathbf{V}$ is the only non-auxetic geometry considered in this study. This geometry, which is meant to mimic a fully-closed tetrachiral system shows a Poisson's ratio of almost zero. This structure is characterised by high levels of shear deformation which results in only the horizontally inclined ligaments deforming (see Figure 5b). Edge effects also appear to play a minimal role on the deformation of this system with the measured Poisson's ratio at the boundaries being almost identical to that measured in the centre. This is extremely surprising since a previous study on area perforated equivalents of these systems indicated that this system should exhibit a significantly negative Poisson's ratio under the loading conditions of the tensile machine due to the restriction of shear deformation by the clamps favouring rotational deformation of the square unit [16]. However, although this effect is responsible for the minute negative Poisson's ratio observed (less than -0.05), the system still deforms predominantly through shear-directed deformation. This is most probably due to the fact that in the slit perforated system is in its fully-closed state since at extremely high strains the Poisson's ratio of the system gradually decreases with the system exhibiting a slightly negative Poisson's ratio as the restriction on shear deformation increases on increasing strain. The last geometry in this set, Architecture VI, shows a Poisson's ratio of $c a$. -0.3 from the central regions of the simulations and experimental tests and a slightly less negative value of ca. -0.2 when considering the entire unit cell. These values show that, like Architecture $\mathbf{V}$, the influence of edge effects on the Poisson's ratio is minimal and that the targeted tri-chiral honeycomb-like deformation was reached. 


\section{$\underline{\text { Set } 3}$}

On the other hand, contrary to Set 2, Set 3 architectures showed the least congruency between the two sets of simulations and the experimental testing (see Figure 6). Unlike, previously discussed architectures, the deformation of these systems was dominated by boundary effects which resulted in significant deviations from the mechanical properties predicted by the corresponding periodic simulations. In fact, in Architecture XI, the nature and positioning of the deformation observed throughout the system made it impossible to measure with a suitable degree of reliability the Poisson's ratio of the central repeating unit of the experimental prototype (see Figure 8).

As one may observe from Figure $6 \mathbf{a}$ and $\mathbf{b}$, in both the periodic and finite simulations, the deformations and the Poisson's ratio changes over tensile strain of the central regions of Architectures VII and VIII are extremely similar. On the other hand, the values obtained from the central regions of the experimental samples show a significantly lower magnitude of negative Poisson's ratio which decreases steadily as strain increases. In addition, the dip and rise of Poisson's ratio seen in the simulations is also not observed in the experimental results, however this could be due to the fact that it occurs over a relatively low initial strain range from which accurate DIC data is extremely difficult to obtain (in fact, only one or two points were obtained at these strains of the central regions). The difference in Poisson's ratio between simulation and experimental results is most probably due to the asymmetric nature of the deformation in the real sample. This asymmetry is most probably a consequence of imperfect cuts resulting in thicker or thinner ligaments which in concert with the extensive boundary effects (see Figure 7) experienced by these systems result in a distorted deformation profile of the central repeating unit. However, despite these problems the central regions of the experimental samples still showed an initial negative Poisson's ratio of $c a$. -3 which became less negative with increasing strain and exhibit all the hallmarks of the re-entrancy. 

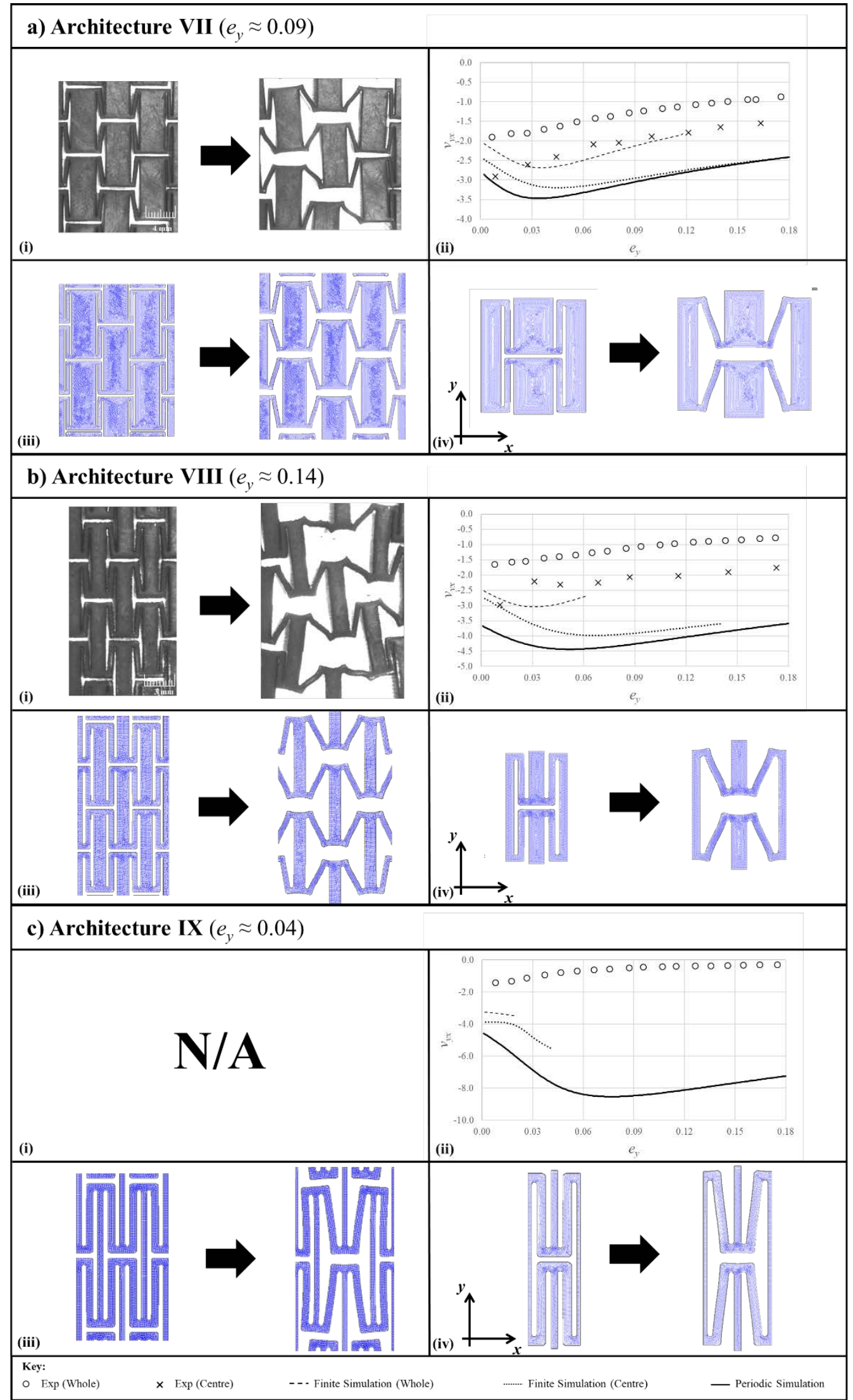

Figure 6: Diagram showing the central regions of the Set 3 (i) experimental and (iii) corresponding Finite Element simulation and (iv) the full periodic simulation for Architectures (a) VII, (b) VII and (c) IX. The engineering Poisson's ratio, $v_{y x}$, vs engineering strain, $e_{y}$, plots for each system up to $18 \%$ strain as measured from the boundary and central regions of each sample are presented in (ii). Architecture IX showed a non- 
uniform deformation in the case of the experimental sample and hence its deformation could not be characterized by that of the central repeating unit (see Figure 6). Videos of experimental tests and simulations for these systems are presented in ANIM19-28.

The nature of the boundary effects experienced by these two architectures are highlighted in Figure 7a and shown in ANIM19 and ANIM22. Unlike Architectures I-VI, these systems do not show a gradual deformation gradient from the regions in proximity to the clamps right up to the central areas of the sample. Instead, the deformation travels through the system in the form of four distinct veins originating from the corners which meet at the centre of the system, with the other repeating units remaining almost completely undeformed. This behaviour, which was mirrored in the corresponding simulations on the finite system (see ANIMS20 and 23), is highly unusual and is reminiscent of a crack propagating through a system. The reason behind this phenomenon becomes apparent once the stress-strain plots of the periodic simulations for these architectures and the previous ones are analysed.

(a)
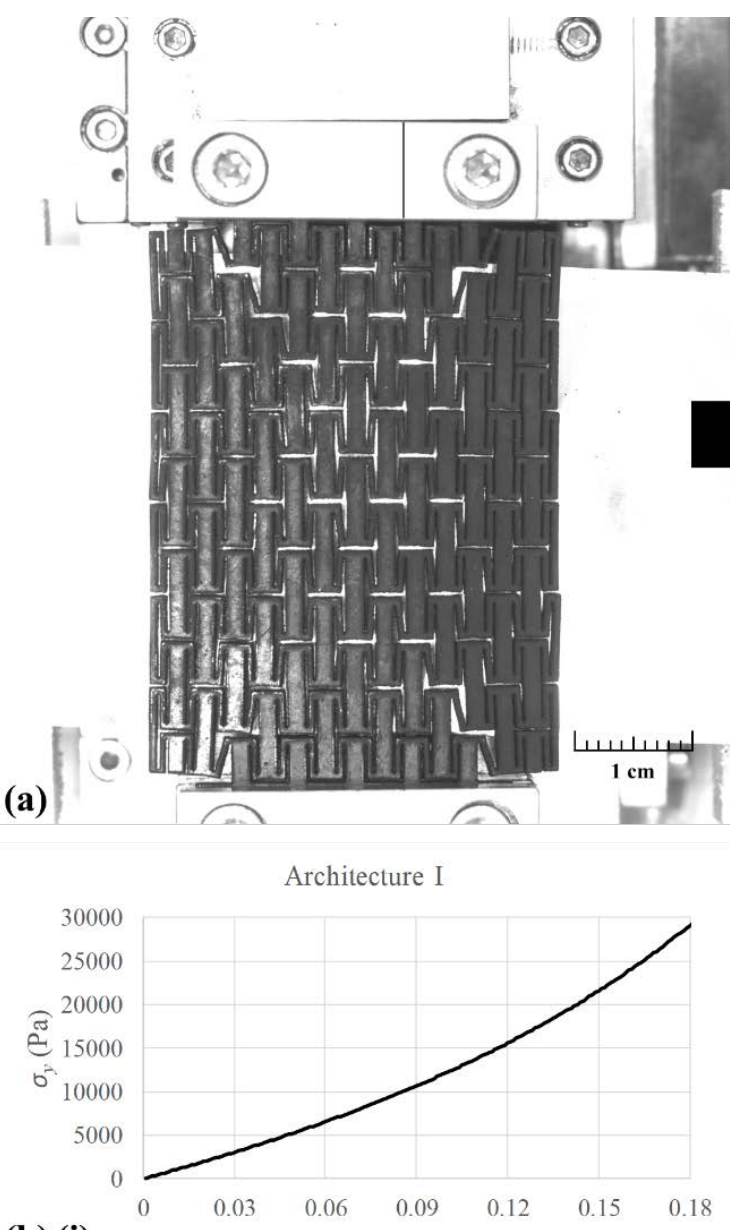

(b) (i)

Figure 7: a) Figure showing the deformation path of Architecture VIII and b) plots showing the nominal stress, $\sigma_{y}$, vs engineering strain, $e_{y}$, for the Architectures i) I and ii) VIII as obtained from the periodic planar finite element simulations. 
As one may observe from Figure 7b, the stress-strain plots for i) Architecture I and ii) Architecture VIII show contrasting trends. These trends are mirrored for Architectures IIIV X-XII and Architectures VII, IX respectively (all plots are presented in Supplementary Information Section 5). While the former starts from a relatively low Young's modulus which increases with increasing strain, the Young's modulus of the latter gradually decreases. Therefore, while in Architecture I, the increasing stiffness on increasing strain results in deformation gradient since the more deformed parts of the system are stiffer than the less deformed regions, the opposite occurs in Architecture VIII, since the most deformed parts are more amenable to deforming further than the non-deformed parts. This results in a deformation behaviour which is analogous to material yielding where deformation is localized at a weak point upon increasing strain. However, unlike plastic or metallic yield, this process is purely mechanical rather than of a material origin, i.e. it is a result of the metamaterial geometry, and thus is entirely reversible. Moreover, despite the lack of deformation from the majority of the repeating units within the system, the large auxetic behaviour imparted by the few deforming repeating unit within the deformation 'veins' still results in an overall highly negative Poisson's ratio. However, this effect is expected to be heavily suppressed in the case of systems with a very large number of repeating units.

Architecture IX was also predicted by the FE simulations to deform in a similar manner to Architectures VII and VIII, as shown in Figure 6c. However, unlike these structures, the deformation of this system is entirely dominated by out-of-plane bending, rather than in-plane re-entrancy as shown in Figure 8, with the deformation occurring sequentially row by row. This is particularly visible from the side view image shown in Figure $\mathbf{8 b}$ (the full video of the tensile test from this angle is provided in ANIM37). This deformation behaviour is the result of the inherent relatively large in-plane effective Young's modulus associated with quasi-fully closed re-entrant honeycombs [52] and corresponding slit perforated systems [51]. Unlike the mechanisms attained by the architectures in the previous sets, the other competing mechanisms with in-plane re-entrancy are not only material stretching in this case, but also out-of-plane bending, which is particularly favoured in fully closed re-entrant geometries with very thin ligaments. Because of this characteristic, unless the system has a considerable out-of-plane thickness, it will deform in the manner shown in the 3D periodic FE simulation in Figure 8c (see Supplementary Information Section 4 for more details on this simulation), where instead of opening up, the system undergoes asymmetric out-of-plane flexure of the inclined 'ribs'. This deformation mechanism is not conducive to generating a large in-plane negative Poisson's 
ratio and therefore the resultant experimentally measured Poisson's ratio was far lower in magnitude than that predicted by the simulations. Out-of-plane deformation, however, may be restricted if a thicker sheet is used to produce the perforated system or by designing the system with a thicker vertical set of ligaments (similar to Architectures VII and VIII), since the effective stiffness of this mechanism is heavily influenced by these parameters and therefore one should always be mindful of this relationship when designing re-entrant systems from perforated sheets.
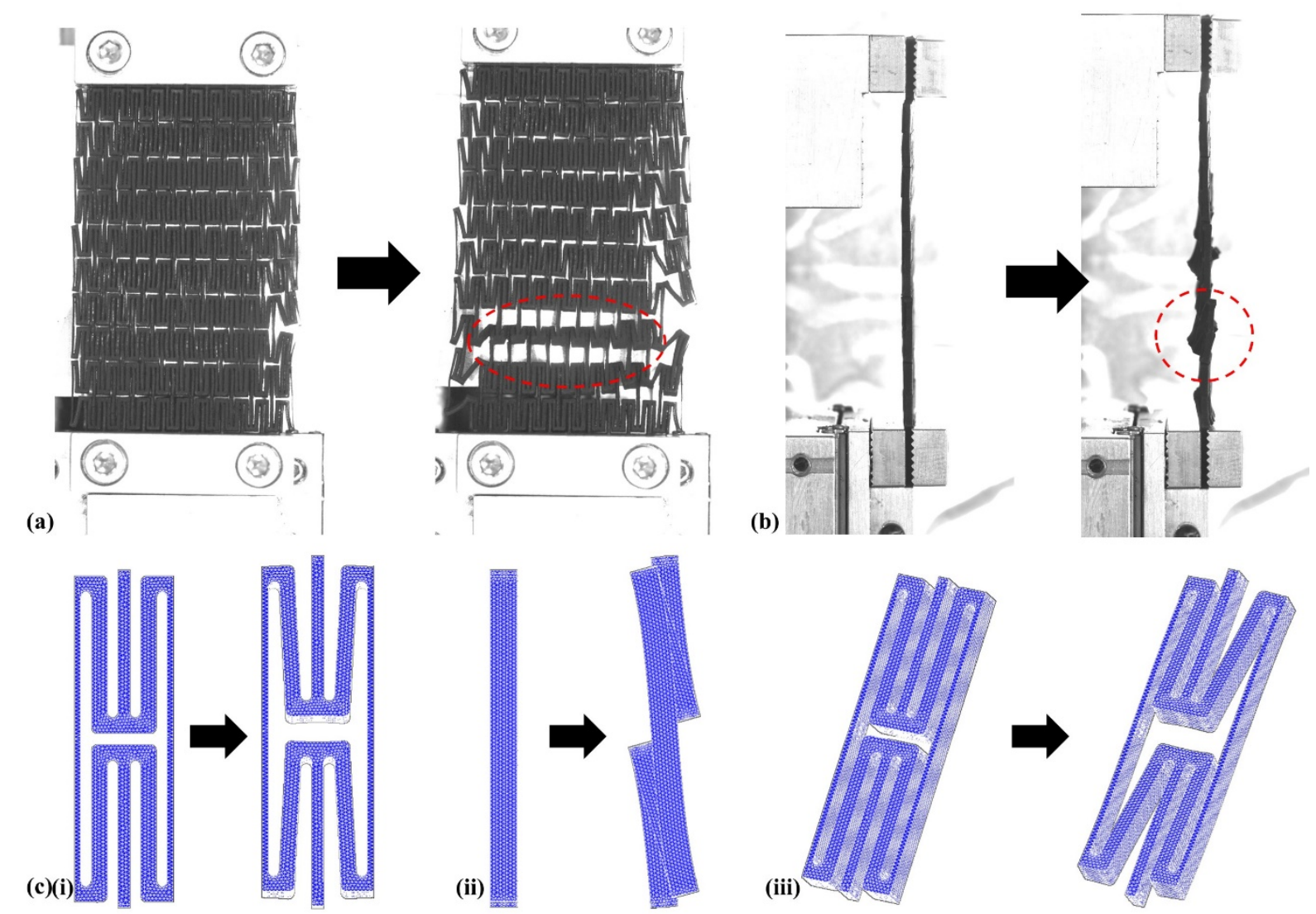

Figure 8: Figures showing the a) front view and b) side view deformation (before and after) of Architecture IX and the red circles indicate a row of repeating units deforming out-of-plane. This deformation is explained in c) where the same behaviour observed from a 3D non-linear simulation on an in-plane periodic repeating unit made from SOLID elements from different viewpoints is shown. Viewpoints c(i) and c(ii) correspond to the experimental (a) and (b).

\section{$\underline{\text { Set } 4}$}

The final set of architectures comprised systems designed to mimic the anti-tetrachiral mechanism. Architecture $\mathbf{X}$, shown in Figure 9a, showed a regular anti-tetrachiral-like deformation profile in both experimental and simulation results. The experimental system showed a constant central Poisson's ratio of $c a$. -0.85 , which was slightly lower in magnitude than the -1 value predicted by the numerical simulations. 

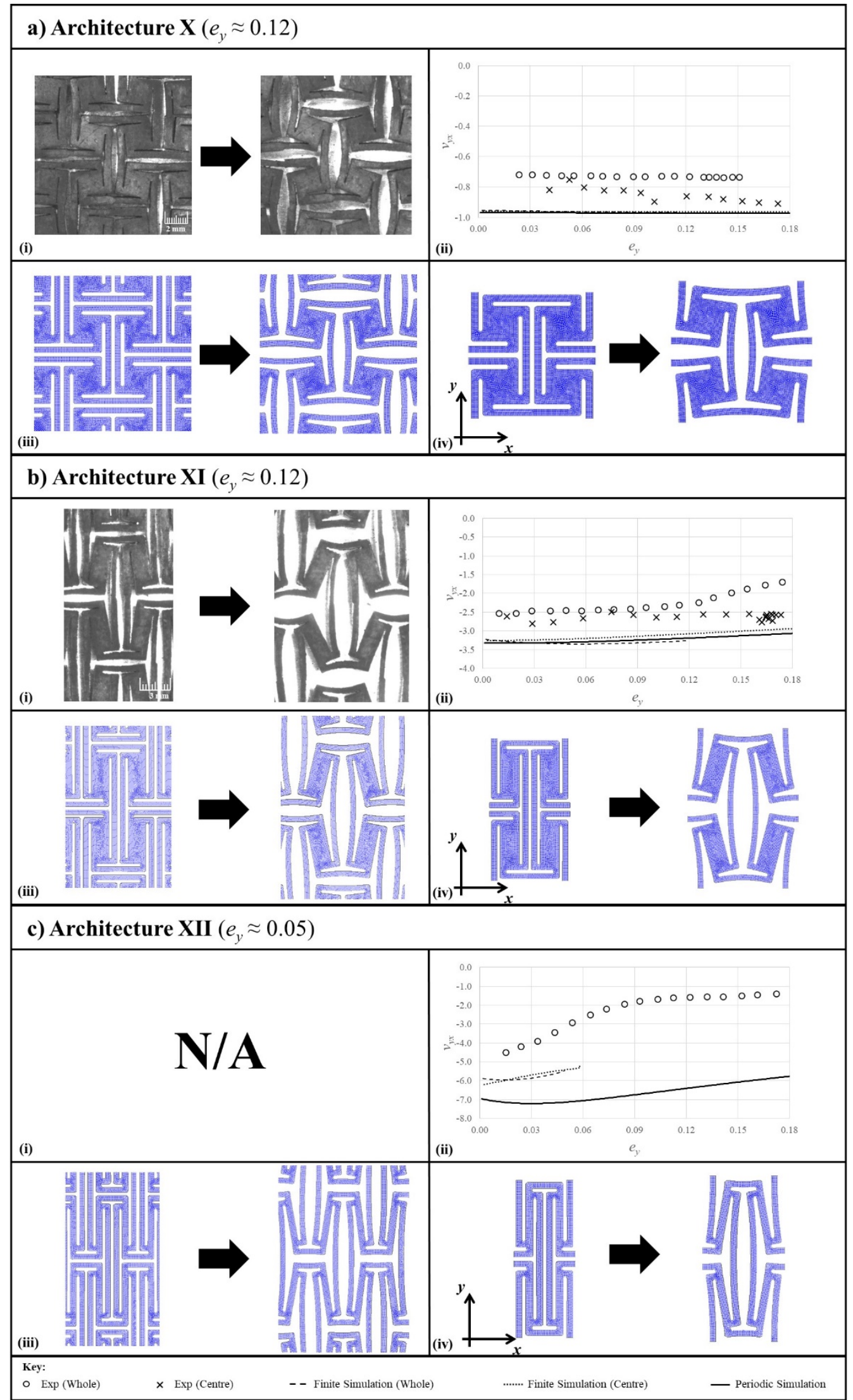

Figure 9: Diagram showing the central regions of the Set 4 (i) experimental and (iii) corresponding Finite Element simulation and (iv) the full periodic simulation for Architectures (a) X, (b) XI and (c) XII. The engineering Poisson's ratio, $v_{y x}$, vs engineering strain, $e_{y}$, plots for each system up to $18 \%$ strain as measured from the boundary and central regions of each sample are presented in (ii). Architecture $X$ also showed a 
non-uniform deformation for the experimental sample as shown in Figure 9c. Videos of experimental tests and simulations for these systems are presented in ANIM29-36.

This similarity between experiment and simulations was also observed for Architecture XI (see Figure 9b). While, the simulations predicted a negative Poisson's ratio of $c a$. -3.3 which gradually lowers to $c a$. -2.9 over an $18 \%$ strain range, the experimental results showed a slightly less negative Poisson's ratio of $c a$. -2.7 which was retained over the same level of tensile strain. This result is extremely significant since it shows that it is possible to achieve giant auxetic behaviour [28] which is retained over a high strain range through the introduction of this perforation pattern. The other architectures studied here with the potential to exhibit Poisson's ratios lower than -1 either possessed a low strain threshold (Architecture III) or else were significantly affected by boundary effects (Architectures VI and VII) or out-of-plane (Architecture IX) deformations. However, Architecture XI, does not appear to suffer from any of these drawbacks and was also observed to be relatively unaffected by edge effects with both the finite simulation and experimental sample showing a nearly identical central and overall Poisson's ratio (see also Figure 10a).

(a)

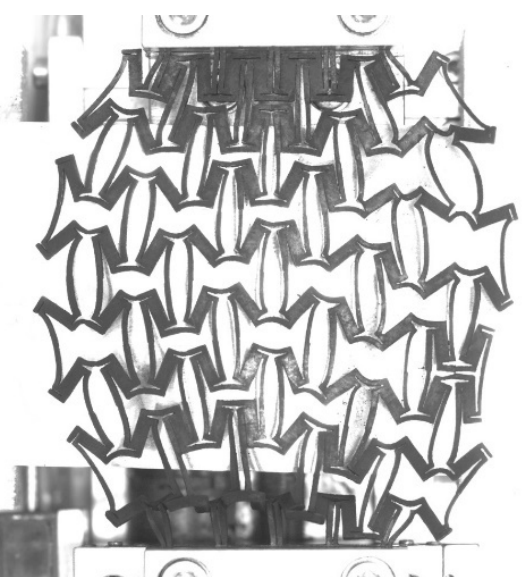

(b)

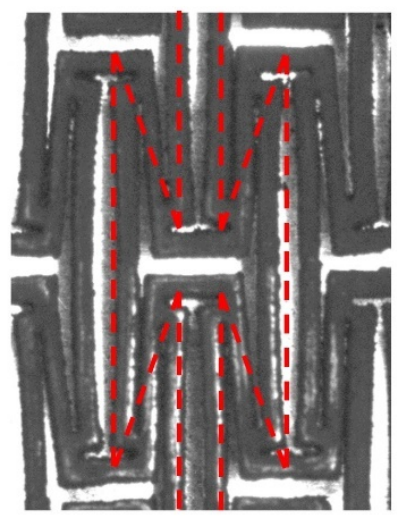

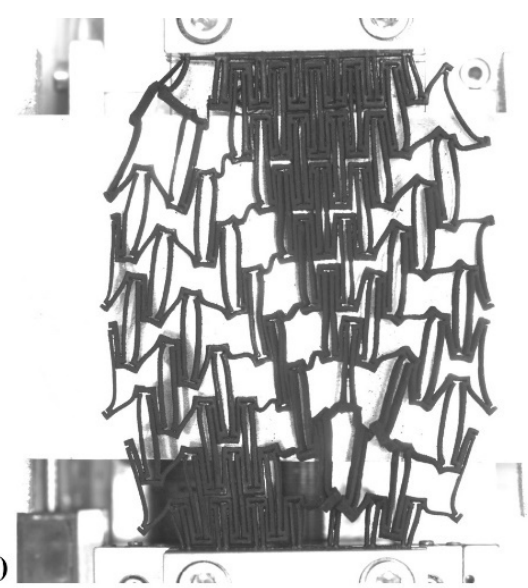

Figure 10: (a) Architecture XI and (c) Architecture XII at $e_{y}$ (overall) $=0.18$. (b) Figure showing the reentrant-like geometric configuration of Architecture XII (at $\left.e_{y}=0\right)$ as a result of the extremely thin 'rotating units' which appear as ligaments.

The final architecture in this set is an extreme version of the previous geometry where the resultant perforated system is made up of ligament-like rather than rectangular rotating units. Due to the large difference between the lengths of vertical and horizontal ligaments, this system is expected to possess a highly negative Poisson's ratio, however this is not the case for the experimental sample. The reason for this is apparent from Figure 10b. The parameters chosen to design Architecture XII have resulted in not only the two pairs of ligaments having an extremely large difference in length, but also in the rotating 'rectangle' to which the main ligaments are attached being reduced to a ligament itself with a thickness comparable to the 
proper ligaments. This results in a tendency to undergo deformations similar to those observed for the Set 3 architectures. As one may observe from Figure 10c, the deformation seems to travel in distinct veins similar to that shown in Figure 7a for Architecture VIII, although in a less symmetrical manner. Moreover, certain repeating units also deformed out-of-plane in a fashion similar to that observed for Architecture IX, but in a far less uniform order (see ANIM38). This finding highlights the delicate balance that one must keep in mind when designing systems based on this perforation type - having ligaments with large differences in lengths will result in giant auxetic behaviour only if the central rotating unit is structurally stiff enough to remain rigid and behave as a rotating unit rather than as a ligament itself.

\section{Discussion}

\section{Mechanical Properties}

All of the twelve architectures covered in this study showed the potential to exhibit auxetic behaviour, except Architecture V which showed, as expected, a Poisson's ratio of zero. The experimental Poisson's ratios obtained ranged from 0 to $c a$. -3.5 and were in most cases retained over a significantly high strain range (ca. 18\%). The closest similarity between experimental and simulation results were observed for systems possessing Poisson's ratios between 0 and -1 . This was to be expected since in highly anisotropic systems with Poisson's ratios less than -1 , any defect and/or boundary effect within the system, as well as inaccuracies in the DIC evaluation, is expected to have a more amplified effect on the central and overall Poisson's ratio of the system. However, as evident from the figures, the expected deformation mechanism was still attained in most cases despite the difference in Poisson's ratio magnitude.

In terms of stress-strain behaviour, the systems in Set 1, showed the highest effective Young's moduli while Architectures V and VI showed the lowest (plots are presented in Supplementary Information Section 6). This was to be expected since mechanical metamaterials made up of only rotating units are typically much stiffer than systems with ligaments. In addition, as expected, regular geometries with Poisson's ratios close to -1 showed lower effective Young's moduli when compared with their irregular counterparts with highly negative Poisson's ratios, i.e. Architecture I vs II and III, and Architecture X vs XI. 


\section{Boundary Effects}

The influence of edge effects on the deformation behaviour of these systems can be described in two parts. For the architectures in Set 1,2 and 4, the deformation occurs in the characteristic manner; the repeating units close to the clamped regions deform the least while those in the centre of system deform the most. This results in a deformation gradient which extends throughout the entire finite system and depending on the type of mechanism by which the system deforms and the degrees of freedom it possesses, the maximum deformation occurs either at the centre of the system or at the central edges of the system.

On the other hand, in the architectures in Set 3, the deformation is not distributed throughout the system but, rather, concentrated along lines of repeating units with the other cells remaining almost completely undeformed, as shown in Figure 7a. This behaviour was extremely surprising and is probably the result of the decreasing Young's modulus of this geometry upon increasing strain (see Figure 7b(ii) and Supplementary Information Section 6). This deformation pattern also suggests that simulating such systems in the form of periodic unit cells will only give an indication of the localized deformation of the repeating unit within the deformation 'vein' rather than a mean picture of the overall deformation. These results indicate that by analysing the stress-strain plots obtained from simulations using periodic boundary conditions one may possibly be able to predict whether a finite system behaves in this manner or else in the 'normal' form such as that shown for the other architectures, where the Young's modulus increases upon increasing strain.

The localized propagation of deformation in Set 3 systems also opens up a number of exciting possibilities. The deformation pathway shown in Figure 7a assumes a criss-cross pattern primarily due to the clamping method used. However, it is envisaged that by using strategically placed stiff regions within the system or applying a point strain on the boundary of the system rather than on the entire edge, one may be able to control the propagation of deformation throughout the system, although further studies are required to fully understand this phenomenon and how to control it effectively.

\section{Out-of-plane Deformation}

All of the architectures presented here were cut from a $1 \mathrm{~mm}$ thick rubber sheet. Given that the thickness of the joints/ligaments was defined by the perforation separation parameter, $s$, which 
was set to $0.4 \mathrm{~mm}$, all systems had a joint/ligament out-of-plane to in-plane thickness ratio of at least 2.5. This should ensure that in-plane deformation is favoured over out-of-plane, as was found out to be the case for the majority of systems studied here. In fact, except for Architectures IX and XII, all systems were characterised by predominantly in-plane deformation modes. Even, in the cases of Architectures II and III, where a small degree of out-of-plane deformation was observed at higher strains, this only occurred once the in-plane rotating unit mechanism had reached its fully-opened form and could not function any longer on increasing strain, thereby justifying the use of FE simulations under plane-stress conditions to analyse these systems. On the other hand, despite the larger relative out-of-plane thickness in comparison to in-plane ligament thickness, the out-of-plane deformations still dominated Architecture IX, and to a lesser extent, Architecture XII. This behaviour may be attributed to the propensity of quasi-fully-closed re-entrant systems to deform in the manner shown in Figure 8c. This indicates that when working with architectures similar to these, it is necessary to use a thicker material in order to ensure that in-plane re-entrancy is the predominant deformation mode.

\section{Defects}

The fabrication method chosen to produce these architectures in general produced the desired architectures to a high degree of accuracy despite the relatively small sizes of the perforation patterns. Although there were some minute variations in the perforation thicknesses (see Figure 2b), in all cases the overall desired geometry was attained and in none of the experimental samples studied here can the observed deformation be described primarily in terms of a disordered geometry or localized defect effects. Despite this, in some cases, even presence of a small number of defects may be responsible for certain differences found between the finite simulations and experimental tests. For example, previous studies on disorder auxetic systems have shown that mechanisms with limited degrees of freedom such as rotating unit modes [29] and hexachiral honeycombs [59], a small increase in the in-plane thickness of even one joint or ligament as a result of an imperfection during the production method, can lead to a slight, but noticeable, reduction in auxeticity similar to what was observed for Architectures I-IV. Moreover, as one may observe from Figure 9a(i), architectures with more complex perforation patterns such as Architectures X-XII, possessed a larger percentage of unequally thick ligaments than the other architectures, quite possibly due to the complicated laser 
pathway which must be undertaken rendering the architecture more susceptible to manufacturing problems such as residual stress effects. However, despite this increased number of imperfections, the deformation of these systems was not observed to be significantly affected by this factor, indicating that an extremely high-resolution fabrication method is not necessarily required in order to obtain the desired deformation in these metamaterial systems.

\section{Stretchability and Surface Coverage}

In the previous sections, the results were presented up to a tensile strain of $18 \%$, which roughly corresponds to the allowed $10 \mathrm{~mm}$ extension by the micro tensile loader. All systems reached this strain threshold without undergoing any irreversible deformations, however, this was by no means the maximum permissible strain level which these systems can tolerate. Although Architectures I-IV are expected to have a relatively small strain threshold due to geometric limitations of the mechanisms through which they deform, the other architectures possess the ability to tolerate far higher tensile strain thresholds whilst retaining their auxetic properties as shown in Figure 11a. In fact, Architectures VI and XI could be reversibly stretched as much as $140 \%$ and $60 \%$ strain respectively.

As typical of auxetic systems, these architectures have the ability to undergo synclastic curvature (see Figure 11b). This property makes them ideal for surface coverage of curved and/or irregular surfaces, while their high stretchability coupled with large auxetic behaviour also ensures that the final surface area covered by the stretched systems is much larger than that initially covered by the undeformed perforated architecture. 
(a)(i)
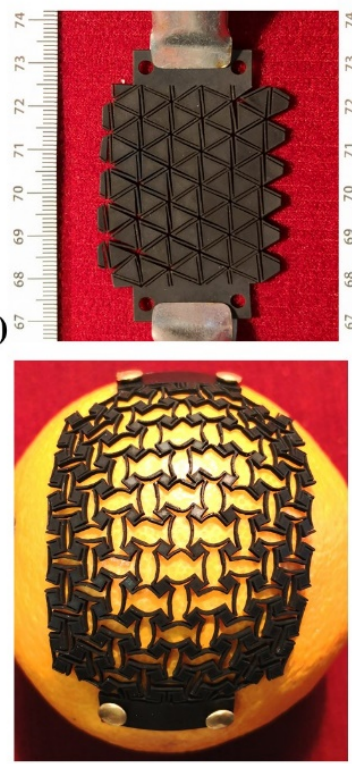

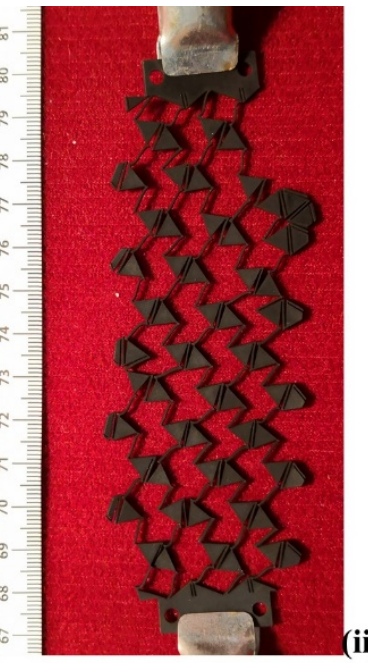

(ii)
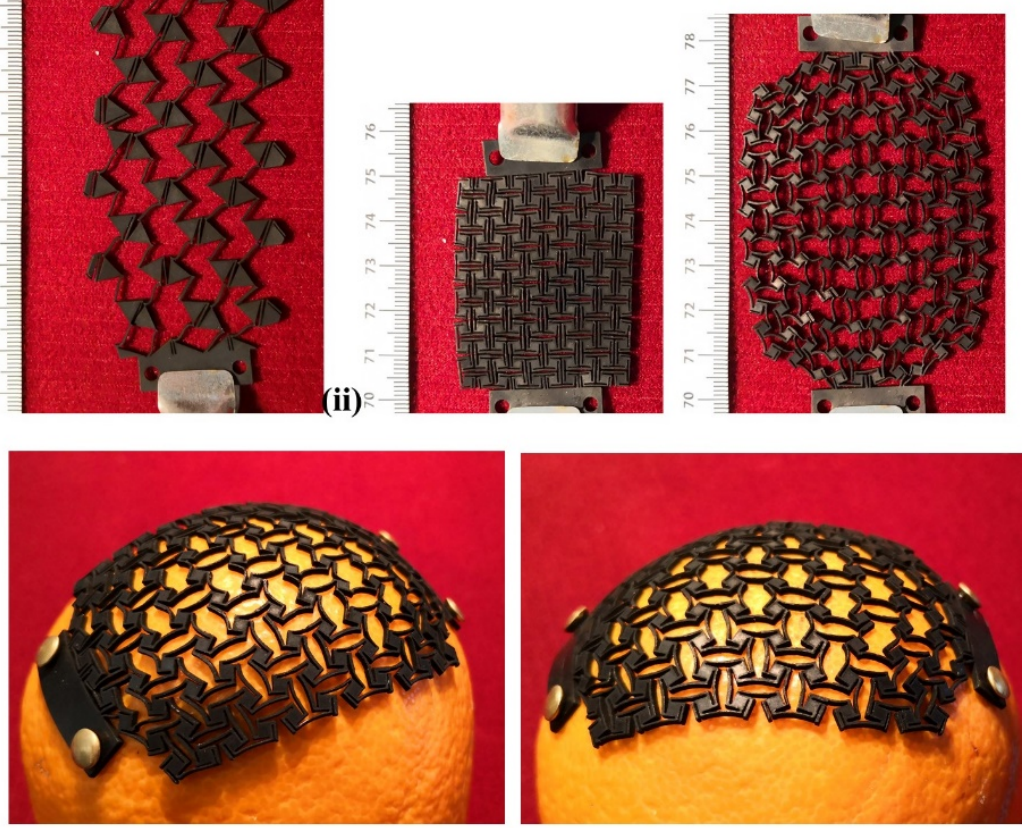

Figure 11: a) Diagrams showing i) Architecture VI and ii) Architecture $X$ stretched up to $140 \%$ and $60 \%$ respectively and b) Architecture $X$ stretched over a large rough spherical surface.

The significance of this study extends beyond the twelve architectures investigated here. In this work, we have shown that it is possible to design with a high degree of accuracy, a range of micro-structured mechanical metamaterial architectures with tailor made auxetic characteristics using direct laser cutting. Some of these architectures have also been experimentally shown to possess the ability to exhibit Poisson's ratios with negative values lower than -3 over a considerable strain range which is extremely significant since achieving such low values is generally considered to be extremely difficult in thin $2 \mathrm{D}$ systems due to the propensity of highly anisotropic geometries to undergo out-of-plane deformations which limit the effectiveness of their in-plane deformations. However, in this study we have shown that these difficulties are not insurmountable and, provided that the right balance is struck between the out-of-plane thickness of the sheet and in-plane dimensions of the perforation pattern, one may still obtain a thin 2D metamaterial which elicits a desired mechanical response to tensile loading.

Finally, it is important to mention that the geometries tested here represent merely the tip of the iceberg in terms of possible metamaterial geometries which may be produced using this method. As amply shown in previous theoretical and numerical studies on 2D auxetic systems, 
rotating quadrilateral systems such as Architectures I-III could be designed in a myriad of forms and sizes, specifically made to exhibit a desired Poisson's ratio ranging from highly positive values to giant auxetic behaviour. Moreover, Architectures IV-VI represent only the regular versions of chiral metamaterials, other systems possessing unequally sized pairs ligaments with the potential to exhibit highly anisotropic behaviour could also be constructed using a similar methodology. Furthermore, besides the rotating quadrilateral, chiral, re-entrant and anti-chiral systems investigated here, other 2D metamaterial mechanisms such as rotating triangle and mixed shape rotating systems could also be potentially designed through this method, as well as hybrid geometries made from a combination of two or more of the designs studied here. The surprising properties observed for the re-entrant-like architectures (Architectures VII-IX) also open up new avenues for exploitation of these systems since it suggests the possibility of directing deformation paths along specific pathways in a uniform repeating geometry. This finding potentially opens up a wide range of novel potential applications, particularly for deployment of these systems in the field of sensors since the deformation pathway appears to be heavily influenced by the type and position of loading. In addition, the high stretchability and surface coverage of these systems coupled with the inherent scalability characteristic of all auxetic geometries, makes them particularly ideal for implementation in applications where these properties are especially desired such as flexible circuits[60] and skin grafts[28]. Overall, the application of direct laser cutting opens up a nearly endless list of possibilities to facilely fabricate thin 2D mechanical metamaterials exhibiting a wide range of deformation and mechanical responses, both rapidly and accurately.

\section{Conclusion}

In conclusion, in this work twelve perforated architectures made from microstructural cuts based on six distinct types of auxetic metamaterial geometries were designed and tested using a laser cutter and a micro-tensile loading device. The deformation behaviour and Poisson's ratios of these systems were evaluated using DIC and Finite Element simulations which showed that these systems have the ability to exhibit highly tuneable Poisson's ratios ranging from zero to highly negative values lower than -3 . These versatile perforated systems have also been shown to be extremely stretchable, with many architectures retaining their auxeticity over a very large strain range, which could make these systems particularly useful for implementation in applications in the biomedical, sensing and electronics fields. It is hoped that this work will 
also inspire further studies in the field of two-dimensional metamaterial sheets, particularly for the design of bespoke micro and nano-structured systems.

\section{Acknowledgements}

The authors would like to thank Prof. Alfonso Castrejon-Pita for his useful advice and for providing access to the laser cutter used to fabricate the experimental prototypes. L.M. and A.M.K. would like to thank the Royal Society for the Commonwealth Science Conference Follow-On Mentoring Grant $2017 \mathrm{CSC} \backslash \mathrm{R} 1 \backslash 170018$ which enabled this collaboration.

\section{References}

[1] Evans, K.E., Nkansah, M.A., Hutchinson, J., Rogers SC. Molecular Network Design. Nature 1991;353:124.

[2] Scarpa F, Ciffo LG, Yates JR. Dynamic properties of high structural integrity auxetic open cell foam. Smart Mater Struct 2004;13:49-56. doi:10.1088/0964-1726/13/1/006.

[3] Scarpa F, Giacomin JA, Bezazi A, Bullough WA. Dynamic behavior and damping capacity of auxetic foam pads. Smart Mater Struct 2006:61690T. doi:10.1117/12.658453.

[4] Bhullar SK, Ko J, Ahmed F, Jun MBG. Design and Fabrication of Stent with Negative Poisson’s Ratio. Int J Mech Aerospace, Ind Mechatronics Eng 2014;8:462-8.

[5] Wu W, Song X, Liang J, Xia R, Qian G, Fang D. Mechanical properties of antitetrachiral auxetic stents. Compos Struct 2018;185:381-92. doi:10.1016/j.compstruct.2017.11.048.

[6] Panico M, Langella C, Santulli C. Development of a Biomedical Neckbrace through Tailored Auxetic Shapes. Ital J Sci Eng 2017;1:105-17.

[7] Kapnisi M, Mansfield C, Marijon C, Guex AG, Perbellini F, Bardi I, et al. Auxetic Cardiac Patches with Tunable Mechanical and Conductive Properties toward Treating Myocardial Infarction. Adv Funct Mater 2018;1800618. doi:10.1002/adfm.201800618.

[8] Duncan O, Shepherd T, Moroney C, Id LF, Venkatraman PD, Winwood K, et al. Review of Auxetic Materials for Sports Applications : Expanding Options in Comfort 
and Protection. Appl Sci 2018;8. doi:10.3390/app8060941.

[9] Olympio KR, Gandhi F. Zero Poisson's ratio cellular honeycombs for flex skins undergoing one-dimensional morphing. J Intell Mater Syst Struct 2010;21:1737-53.

[10] Airoldi A, Bettini P, Panichelli P, Sala G. Chiral topologies for composite morphing structures - Part II: Novel configurations and technological processes. Phys Status Solidi Basic Res 2015;252:1446-54. doi:10.1002/pssb.201584263.

[11] Li Q, Kuang Y, Zhu M. Auxetic piezoelectric energy harvesters for increased electric power output. AIP Adv 2017;7:015104. doi:10.1063/1.4974310.

[12] Groeger D, Steimle J. LASEC : Instant Fabrication of Stretchable Circuits Using a Laser Cutter. CHI19, 2019.

[13] Zheng X, Lee H, Weisgraber TH, Shusteff M, DeOtte J, Duoss EB, et al. Ultralight, Ultrastiff Mechanical Metamaterials. Science (80- ) 2014;344:1373-8.

[14] Jiang BY, Li Y. 3D Printed Chiral Cellular Solids with Ampli fi ed Auxetic Effects Due to Elevated Internal Rotation. Adv Eng Mater 2017;19:1600609. doi:10.1002/adem.201600609.

[15] Shen J, Zhou S, Huang X, Xie YM. Simple cubic three-dimensional auxetic metamaterials 2014;8:1-8. doi:10.1002/pssb.201451304.

[16] Mizzi L, Mahdi EM, Titov K, Gatt R, Attard D, Evans KE, et al. Mechanical metamaterials with star-shaped pores exhibiting negative and zero Poisson's ratio. Mater Des 2018;146:28-37.

[17] Farrugia P, Gatt R, Grima JN. A Novel Three-Dimensional Anti-Tetrachiral Honeycomb. Phys Status Solidi 2019;256:1800473. doi:10.1002/pssb.201800473.

[18] Shim J, Shan S, Košmrlj A, Kang SH, Chen ER, Weaver JC, et al. Harnessing instabilities for design of soft reconfigurable auxetic/chiral materials. Soft Matter 2013;9:8198-202. doi:10.1039/c3sm51148k.

[19] Lee J, Singer JP, Thomas EL. Micro- / Nanostructured Mechanical Metamaterials 2012:4782-810. doi:10.1002/adma.201201644.

[20] Fozdar DY, Soman P, Lee JW, Han LH, Chen S. Three-dimensional polymer constructs exhibiting a tunable negative poisson's ratio. Adv Funct Mater 
2011;21:2712-20. doi:10.1002/adfm.201002022.

[21] Bückmann T, Stenger N, Kadic M, Kaschke J, Frölich A, Kennerknecht T, et al. Tailored 3D Mechanical Metamaterials Made by Dip-in Direct-Laser-Writing Optical Lithography. Adv Mater 2012:2710-4. doi:10.1002/adma.201200584.

[22] Bertoldi BK, Reis PM, Willshaw S, Mullin T. Negative Poisson's Ratio Behavior Induced by an Elastic Instability. Adv Funct Mater 2009:1-6. doi:10.1002/adma.200901956.

[23] Grima BJN, Gatt R. Perforated Sheets Exhibiting Negative Poisson's Ratios. Adv Eng Mater 2010:460-4. doi:10.1002/adem.201000005.

[24] Grima JN, Gatt R, Ellul B, Chetcuti E. Auxetic behaviour in non-crystalline materials having star or triangular shaped perforations. J Non Cryst Solids 2010;356:1980-7. doi:10.1016/j.jnoncrysol.2010.05.074.

[25] Taylor M, Francesconi L, Gerendás M, Shanian A, Carson C, Bertoldi K. Low porosity metallic periodic structures with negative Poisson's ratio. Adv Mater 2014;26:2365-70.

[26] Slann, A., White, W., Scarpa, F., Boba, K., Farrow I. Cellular plates with auxetic rectangular perforations Cellular plates with auxetic rectangular perforations. Phys Status Solidi 2015;252:1533-9. doi:10.1002/pssb.201451740.

[27] Shan S, Kang SH, Zhao Z, Fang L. Design of planar isotropic negative Poisson 's ratio structures. Extrem Mech Lett 2015;4:96-102. doi:10.1016/j.eml.2015.05.002.

[28] Mizzi L, Azzopardi KM, Attard D, Grima JN, Gatt R. Auxetic metamaterials exhibiting giant negative Poisson's ratios. Phys Status Solidi - Rapid Res Lett 2015;9:425-30. doi:10.1002/pssr.201510178.

[29] Grima JN, Mizzi L, Azzopardi KM, Gatt R. Auxetic Perforated Mechanical Metamaterials with Randomly Oriented Cuts. Adv Mater 2016;28:385-9. doi:10.1002/adma.201503653.

[30] Overvelde JTB, Shan S, Bertoldi K. Compaction Through Buckling in 2D Periodic , Soft and Porous Structures : Effect of Pore Shape 2012:1-6. doi:10.1002/adma.201104395. 
[31] Cho Y, Shin J, Costa A, Ann T, Kunin V, Li J, et al. Engineering the shape and structure of materials by fractal cut 2014;111. doi:10.1073/pnas.1417276111.

[32] Kunin V, Yang S, Cho Y, Deymier P, Srolovitz DJ. Static and dynamic elastic properties of fractal-cut materials. Extrem Mech Lett 2015. doi:10.1016/j.eml.2015.12.003.

[33] Gatt R, Mizzi L, Azzopardi JI, Azzopardi KM, Attard D, Casha A, et al. Hierarchical Auxetic Mechanical Metamaterials. Sci Rep 2015;5:1-6. doi:10.1038/srep08395.

[34] Carta G, Brun M, Baldi A. Design of a porous material with isotropic negative Poisson 's ratio. Mech Mater 2016;97:67-75. doi:10.1016/j.mechmat.2016.02.012.

[35] Florijn B, Coulais C, Hecke M Van. Programmable Mechanical Metamaterials. Phys Rev Lett 2014;113:175503. doi:10.1103/PhysRevLett.113.175503.

[36] Shyu TC, Damasceno PF, Dodd PM, Lamoureux A, Xu L, Shlian M, et al. A kirigami approach to engineering elasticity in nanocomposites through patterned defects. Nat Mater 2015;14:785-90. doi:10.1038/NMAT4327.

[37] Tang Y, Yin J. Design of cut unit geometry in hierarchical kirigami-based auxetic metamaterials for high stretchability and compressibility. Extrem Mech Lett 2017;12:77-85. doi:10.1016/j.eml.2016.07.005.

[38] Bertoldi K, Vitelli V, Christensen J, Hecke M Van. Flexible mechanical metamaterials. Nat Rev 2017;2:17066. doi:10.1038/natrevmats.2017.66.

[39] Rafsanjani A, Bertoldi K. Buckling-Induced Kirigami. Phys Rev Lett 2017;084301:15. doi:10.1103/PhysRevLett.118.084301.

[40] Rafsanjani A, Jin L, Deng B, Bertoldi K. Propagation of pop ups in kirigami shells. Proc Natl Acad Sci 2019. doi:10.1073/pnas.1817763116.

[41] Ning X, Wang X, Zhang Y, Yu X, Choi D, Zheng N, et al. Assembly of Advanced Materials into 3D Functional Structures by Methods Inspired by Origami and Kirigami : A Review. Adv Mater 2018;1800284:1-13. doi:10.1002/admi.201800284.

[42] Grima JN, Evans KE. Auxetic behavior from rotating squares. J Mater Sci Lett 2000;19:1563-5.

[43] Grima JN, Gatt R, Alderson A, Evans KE. On the auxetic properties of 'rotating 
rectangles’ with different connectivity. J Phys Soc Japan 2005;74:2866-7.

[44] Attard D, Manicaro E, Grima JN. On rotating rigid parallelograms and their potential for exhibiting auxetic behaviour 2009;2044:2033-44. doi:10.1002/pssb.200982034.

[45] Wojciechowski KW. Two-dimensional isotropic system with a negative poisson ratio. Phys Lett A 1989;137:60-4. doi:10.1016/0375-9601(89)90971-7.

[46] Prall D, Lakes RS. Properties of a chiral honeycomb with a Poisson's ratio of -1. Int J Mech Sci 1997;39:305-14.

[47] Alderson A, Alderson KL, Attard D, Evans KE, Gatt R, Grima JN, et al. Elastic constants of 3-, 4- and 6-connected chiral and anti-chiral honeycombs subject to uniaxial in-plane loading. Compos Sci Technol 2010;70:1042-8. doi:10.1016/j.compscitech.2009.07.009.

[48] Grima JN. New Auxetic Materials. 2000.

[49] Bacigalupo A, Gambarotta L. Homogenization of periodic hexa- and tetrachiral cellular solids. Compos Struct 2014;116:461-76. doi:10.1016/j.compstruct.2014.05.033.

[50] Alderson A, Alderson KL, Chirima G, Ravirala N, Zied KM. The in-plane linear elastic constants and out-of-plane bending of 3-coordinated ligament and cylinderligament honeycombs. Compos Sci Technol 2010;70:1034-41. doi:10.1016/j.compscitech.2009.07.010.

[51] Mizzi L, Grima JN, Gatt R, Attard D. Analysis of the Deformation Behavior and Mechanical Properties of Slit-Perforated Auxetic Metamaterials. Phys Status Solidi 2019:1800153. doi:10.1002/pssb.201800153.

[52] Masters IG, Evans KE. Models for the elastic deformation of honeycombs. Compos Struct 1996;35:403-22.

[53] Evans KE, Alderson A, Christian FR. Auxetic Two-dimensional Polymer Networks. J Chem Soc Faraday Trans 1995;91:2671-80.

[54] Bezazi A, Scarpa F, Remillat C. A novel centresymmetric honeycomb composite structure. Compos Struct 2005;71:356-64. doi:10.1016/j.compstruct.2005.09.035.

[55] Alderson KL, Alderson A, Smart G, Simkins VR, Davies PJ. Part 1 - Manufacture and 
characterisation Auxetic polypropylene fibres 2002:1-6.

[56] Sigmund O, Torquato S. Design of smart composite materials using topology optimization. Smart Mater Struct 1999;8:365-79. doi:10.1088/0964-1726/8/3/308.

[57] Gatt R, Attard D, Farrugia PS, Azzopardi KM, Mizzi L, Brincat JP, et al. A realistic generic model for anti-tetrachiral systems. Phys Status Solidi Basic Res 2013;250:2012-9. doi:10.1002/pssb.201384246.

[58] Timoschenko S, Goodier JN. Theory of Elasticity. Engineering Societies Monographs; 1951.

[59] Mizzi L, Attard D, Gatt R, Pozniak AA, Wojciechowski KW, Grima JN. Influence of translational disorder on the mechanical properties of hexachiral honeycomb systems. Compos Part B Eng 2015;80:84-91. doi:10.1016/j.compositesb.2015.04.057.

[60] Huang S, Liu Y, Zhao Y, Ren Z, Guo CF. Flexible Electronics : Stretchable Electrodes and Their Future. Adv Funct Mater 2019;1805924:1-15. doi:10.1002/adfm.201805924. 